\title{
Oscillations on the Sun in regions with a vertical magnetic field
}

\section{On the calculation of the sunspot umbral oscillations}

\author{
V. I. Zhukov^ \\ The Central Astronomical Observatory of Russian Academy of Sciences, 65 Pulkovo, 196140 St. Petersburg, Russia \\ e-mail: leapsy@mail.ru (Zhukova Svetlana)
}

Received 17 June 2004 / Accepted 3 December 2004

\begin{abstract}
We calculated the spectrum of oscillations for the model of the sunspot umbra described in Zhukov (2002, A\&A, $386,653)$. The calculations were carried out both by resonant filtering and from the solution of the eigenvalue problem. Both methods yield the same results, thus confirming the concept according to which both 5-min and 3-min oscillations of the umbra represent the same set of $p$-like mode oscillations of the sunspot. The calculated frequencies of the oscillations and corresponding eigenfunctions are consistent with observations.
\end{abstract}

Key words. Sun: sunspots - Sun: oscillations - magnetohydrodynamics (MHD)

\section{Introduction}

The first attempt to understand the nature of 3-min oscillations in umbrae of solar spots, which had been recently discovered, was made by Uchida \& Sakurai (1975). By analogy with 5-min oscillations of the Sun, they suggested that 3-min oscillations are caused by the presence of an Alfvén wave resonator in the umbra. Scheuer \& Thomas (1981) rejected several simplifying assumptions used by Uchida \& Sakurai (1975). However, they were unable to find asymptotics of solutions of the system of MHD equations in deep layers of the umbra. Therefore, in almost all subsequent studies of umbral oscillations, "closed" boundary conditions were used (Thomas \& Scheuer 1982; Zhukov et al. 1987; Wood 1990, 1997; Hasan 1991; Hasan \& Christensen-Dalsgaard 1992; Gore 1997, 1998; Banerjee et al. 2002). Only Cally \& Bogdan (1993) (see also Cally et al. 1994; Bogdan \& Cally 1997; Lites et al. 1998) were able to calculate the spectrum of umbral oscillations for "open" boundary conditions, which provide the possibility for slow magnetoacoustic waves radiation from spots. Note, however, that these calculations were made for a model of the umbra with a polytropic convective zone; the polytropic index was $\mu=3 / 2$.

Along with these studies, in which the spectra of the umbral oscillations were calculated by the determination of eigenvalues, since the early 80 ies some researchers (Žugžda et al. 1983, 1984; Gurman \& Leibacher 1984; Lee \& Yun 1987; Z̆ugžda et al. 1987; Settele et al. 1999, 2001) have developed resonant filter theory, i.e., the theory of filtration of MAG waves by the atmosphere of the umbra. According to this theory, the atmosphere of an umbra is transparent to slow magnetoacoustic

\footnotetext{
* Deceased on December 26th, 2004
}

waves only in narrow bands located within the observed 3-min oscillations.

Up to now, these two views of the nature of 3-min oscillations in the umbra of a solar spot have remained incompatible. It is assumed that the umbra of a spot contains two resonators: one for fast magnetoacoustic waves, almost captured in the photosphere and subphotospheric layers, the other for slow magnetoacoustic waves, almost captured within the region of temperature minimum and the transition zone. Thomas (1984) (see also Bogdan 2000) noted that the main peak in the power spectrum of 3-min oscillations is caused by the presence of a photospheric resonator, while the closely-spaced multiple peaks, sometimes observed in the power spectra of chromospheric oscillations, may be explained by the presence of a chromospheric resonator. Consequently, according to this point of view, the observed spectrum of 3-min oscillations consists of two parts, one of which may be calculated with the use of resonant filtering, i.e., by calculation of the transmission coefficient for a slow magnetoacoustic wave propagating through the atmosphere of an umbra, while the other may be obtained from the calculation of the eigenvalues.

However, despite numerous efforts, the problem of the nature of the umbral oscillations has not yet been convincingly solved (see reviews by Staude 1999; and Bogdan 2000). In our opinion, this is due to the fact that different authors used somewhat different models of the umbra. For example, for deep layers, Cally \& Bogdan (1993) in their study used a polytrope with the index $\mu=3 / 2$, while Settele et al. (1999) developed a semi-empirical model, which made it impossible to compare correctly the obtained results. Additional difficulties were due to the fact that until now asymptotic solutions of the system of MHD equations have been obtained only for isothermal 
(Hasan \& Christensen-Dalsgaard 1992) and polytropic (with polytropic index $\mu=3 / 2$, see Cally \& Bogdan 1993; Bogdan \& Cally 1997) atmospheres. It was only recently that the asymptotic solutions were found for the atmosphere, in which the Alfvén speed in sufficiently deep layers is considerably smaller than the sound speed (Zhukov 2002). This makes it possible to calculate the spectrum of umbral oscillations for a sufficiently realistic model of the umbra.

Here, we calculate the spectrum of 3-min oscillations for the model of the umbra described in Zhukov (2002). For the first time, for the same model of the umbra, the calculations were made both by the method of resonant filtering and by the solution of the eigenvalue problem. This eventually has made it possible to settle the 20-year argument about the correct technique for the analysis of oscillations in the sunspot umbra.

\section{Basic equations}

Here, we use the two-layer model of the umbra, described in Zhukov (2002) (see Appendix A).

The linear oscillations of this two-layer model of the umbra with a homogeneous vertical magnetic field $H_{0} \boldsymbol{e}_{z}$ in the approximation of ideal magnetohydrodynamics are described by the following well-known set of equations (Ferraro \& Plumpton 1958)

$$
\begin{aligned}
& v_{\mathrm{A}}^{2} \frac{\mathrm{d}^{2} v_{x}}{\mathrm{~d} z^{2}}+\left(\omega^{2}-c^{2} k^{2}-v_{\mathrm{A}}^{2} k^{2}\right) v_{x} \\
&+\mathrm{i} k\left(c^{2} \frac{\mathrm{d} v_{z}}{\mathrm{~d} z}-g v_{z}\right)=0, \\
& c^{2} \frac{\mathrm{d}^{2} v_{z}}{\mathrm{~d} z^{2}}-g \gamma \frac{\mathrm{d} v_{z}}{\mathrm{~d} z}+\omega^{2} v_{z} \\
&+\mathrm{i} k\left(c^{2} \frac{\mathrm{d} v_{x}}{\mathrm{~d} z}-g(\gamma-1) v_{x}\right)=0,
\end{aligned}
$$

where $c=(\gamma R T(z))^{1 / 2}$ is the sound speed, $\gamma$ the ratio of specific heats, $g$ the gravity acceleration, which is assumed to be constant $\left(=0.274 \mathrm{~km} \mathrm{~s}^{-2}\right)$ and $v_{\mathrm{A}}=\left(H_{0}^{2} / 4 \pi \rho\right)^{1 / 2}$ the Alfvén speed.

In the derivation of the set of Eqs. (1) and (2), the following dependence of all perturbed quantities on $x$ and $t$ was taken: $\sim \exp [\mathrm{i}(k x+\omega t)]$.

The system (1) and (2) supplemented by the boundary conditions

$$
\left[v_{z}\right]=0, \quad[\boldsymbol{H}]=0, \quad[p]=0 \quad \text { at } \quad z=0
$$

(continuity of the vertical component of velocity, magnetic field and pressure) and conditions of radiation of slow magnetoacoustic waves at infinity $(z \rightarrow \pm \infty)$ completely determines the problem of the sunspot umbral oscillations.

\section{Initial conditions in deep layers of a sunspot umbra}

The central problem in the calculations of the eigenoscillations of a sunspot umbra is related to the selection of the initial conditions for the system (1) and (2) in deep layers of the umbra.
As was shown by Zhukov (2002), in sufficiently deep layers (at $z \rightarrow-\infty$ ), the system (1) and (2) is divided into two independent subsystems. One of them has the following solutions

$$
\begin{aligned}
& v_{x} \sim \sqrt{v_{\mathrm{A}}} \mathrm{e}^{ \pm \mathrm{i} \int^{z} \frac{\omega}{v_{\mathrm{A}}} \mathrm{d} z}, \\
& v_{z} \sim 0 .
\end{aligned}
$$

These solutions represent slow magnetoacoustic waves.

The other system can be reduced to the equation

$$
\begin{aligned}
& c^{2}\left(\omega^{2}-c^{2} k^{2}\right) \frac{\mathrm{d}^{2} v_{z 0}}{\mathrm{~d} z^{2}}+\left[c^{2} k^{2} \frac{\mathrm{d} c^{2}}{\mathrm{~d} z}-g \gamma\right. \\
& \left.\quad \times\left(\omega^{2}-c^{2} k^{2}\right)\right] \frac{\mathrm{d} v_{z 0}}{\mathrm{~d} z}+\left[\left(\omega^{2}-c^{2} k^{2}\right)^{2}+\frac{(\gamma-1) g^{2} k^{2}}{\omega^{2}}\right. \\
& \left.\times\left(\omega^{2}-c^{2} k^{2}\right)-\frac{g k^{4} c^{2}}{\omega^{2}} \frac{\mathrm{d} c^{2}}{\mathrm{~d} z}\right] v_{z 0}=0 .
\end{aligned}
$$

For a linear temperature profile, the solutions of this equation are well known (Nye \& Thomas 1976; Evans \& Roberts 1990).

Thus, for our model, in sufficiently deep layers of the umbra, the asymptotic solutions of the set of Eqs. (1) and (2) have the form

$v_{x} \sim \sqrt{v_{\mathrm{A}}} \mathrm{e}^{ \pm \mathrm{i} \int^{z} \frac{\omega}{v_{\mathrm{A}}} \mathrm{d} z}$

$v_{z} \sim 0$

at $\quad z=z_{\mathrm{am}}$

and

$v_{z} \sim z^{b} \exp (k z) \quad$ at $\quad z=z_{\mathrm{a}}$,

where

$b=\frac{\mu+1}{\gamma} \frac{\omega^{2}}{2 g k}+\frac{g k}{2 \omega^{2}}\left(\mu-\frac{\mu+1}{\gamma}\right)-\frac{1}{2} \mu$.

Here $z_{\mathrm{am}}$ and $z_{\mathrm{a}}$ are depths at which it is possible to use the asymptotic solutions (7)-(9) accordingly.

In order to determine the spectrum of the eigenoscillations of the sunspot umbra one should, in particular, integrate the stiff system of two coupled second-order differential Eqs. (1) and (2), starting from some depth at which the expressions (7)-(9) may be used as the initial conditions. In our study, all calculations were made for $z_{\mathrm{a}}=-150 z_{2}$ and $z_{\mathrm{am}}=-10 z_{2}$, $z_{2}=250 \mathrm{~km}$. This is considered in more detail in Appendix B; here we only note that for the model parameters accepted in our study, $\beta\left(\equiv 8 \pi p / H^{2}\right)=1$, i.e., according the terms introduced in the study of Rosenthal et al. (2002), the magnetic canopy is located in the vicinity of $z=-250 \mathrm{~km}$.

\section{The spectrum of the sunspot umbral oscillations}

In our calculations of the spectrum of umbral oscillations, to derive the solution describing a slow magnetoacoustic wave, we numerically integrated the fourth-order equation for the horizontal component of the velocity (B.1) from $z=-10 z_{2}$ to $z=0$ rather than the system (1) and (2) (see Appendix B). The calculations were carried out for the same model parameters as in the study of Zhukov (2002), with the exception that here we 


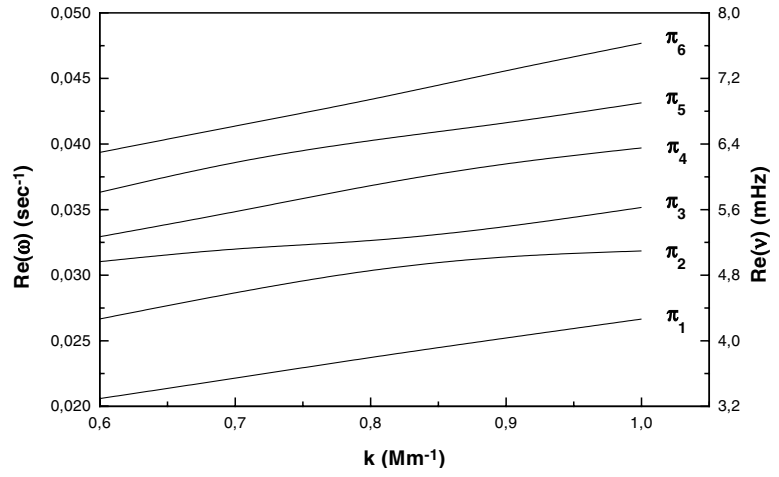

Fig. 1. The real parts of the frequencies of the $\pi$-mode umbral oscillations.

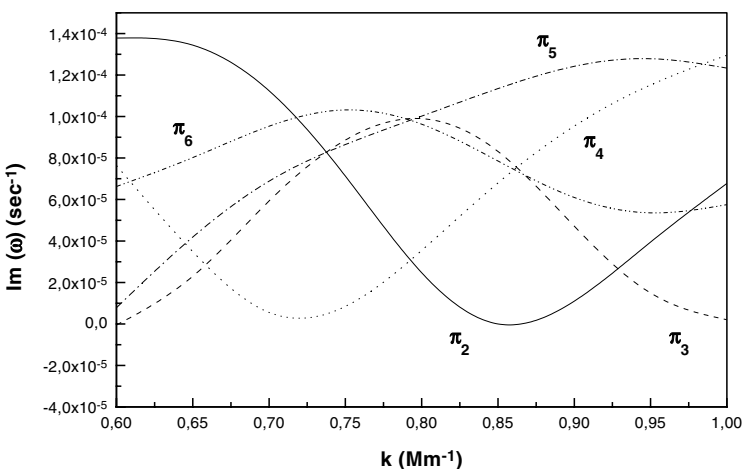

Fig. 2. The imaginary parts of the frequencies of the $\pi$-mode umbral oscillations.

assumed $H_{0}=4000 \mathrm{G}$. Figures 1 and 2 present the results of the calculations. According to the classification introduced by Bogdan \& Cally (1997), these are $\pi$-modes. Figure 2 indicates the presence of several undamped trapped $\pi$-modes, for which $\operatorname{Im}(\omega)=0$. Within the frequency interval $(3 \div 8) \mathrm{mHz}$, no $\sigma$ modes was found.

Figures 3 and 4 present (in arbitrary units) the eigenfunction for $\pi_{6}$-mode, in the calculation of which the amplitude of the solution (9) was assumed to be equal to unity. Since absolute values for both the real and imaginary parts of $v_{z}$ are very large in the transition zone and lower corona, the region $z<0$ is shown separately in Fig. 3b, in order to display the variation of $v_{z}$ in lower umbral layers. Figure 4 presents the variation of both real and imaginary parts of $v_{x}$ as a function of height in the umbra; they clearly display the presence of a slow magnetoacoustic wave ( $s$-wave) in deep umbral layers. In the lower corona $v_{x}$ tends to zero.

\section{The transmission of a slow magnetoacoustic wave through the atmosphere of the sunspot umbra}

It follows from the asymptotics (7)-(9) that in deep layers of the umbra (in the area lower than $z=z_{\mathrm{a}}$ ) only slow magnetoacoustic waves can propagate. Therefore, the analysis of umbral oscillations may be restricted to the case of a slow magnetoacoustic wave propagating through the atmosphere of the sunspot umbra.
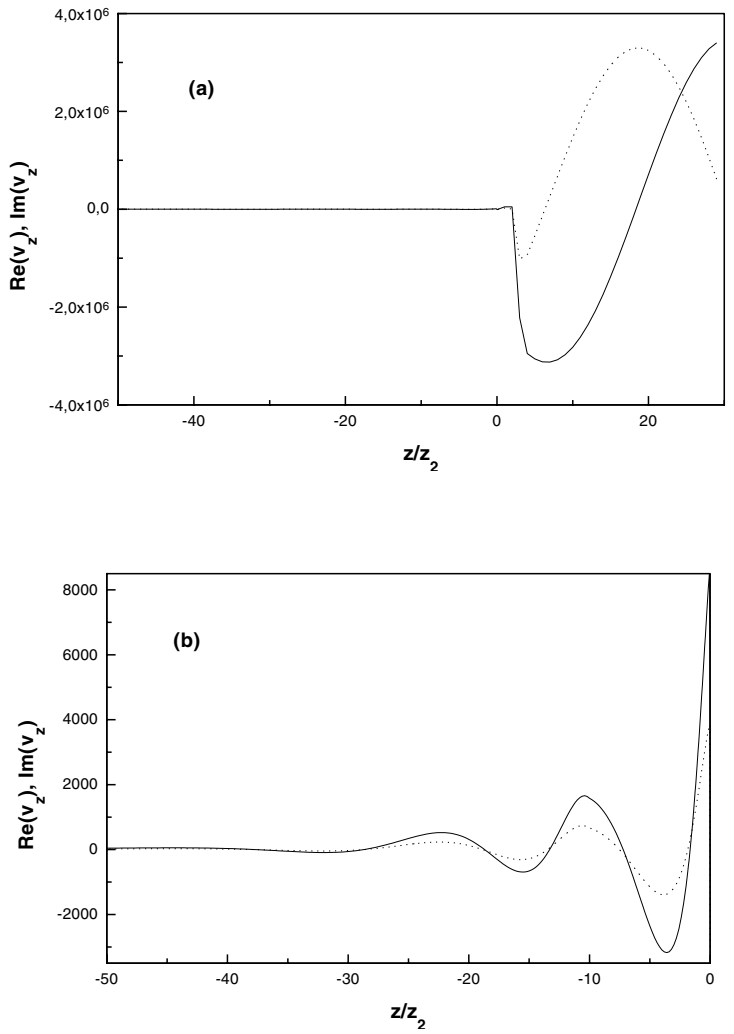

Fig. 3. Real (solid curve) and imaginary (dotted curve) parts of $v_{z}$ for $\pi_{6}$-mode $\left(k=0,8 \mathrm{Mm}^{-1}, H_{0}=4000 \mathrm{G}\right)$.

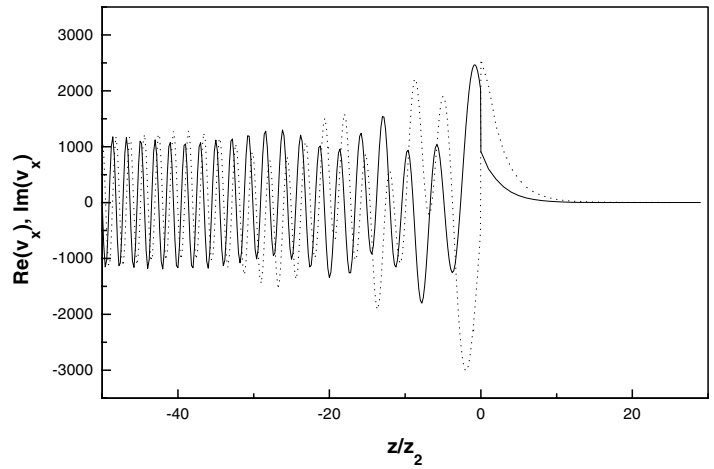

Fig. 4. Real (solid curve) and imaginary (dotted curve) parts of $v_{x}$ for $\pi_{6}$-mode $\left(k=0,8 \mathrm{Mm}^{-1}, H_{0}=4000 \mathrm{G}\right)$.

Let us assume that a fast magnetoacoustic (in fact, acoustic) wave is specified, propagating upward at a sufficiently large depth $z=z_{i}$, where fast and slow waves do not interact (in our notation, below the $z=z_{\mathrm{m}}$ layer). Further, taking into account the boundary conditions in the corona (for $z \rightarrow \infty$ ), it is possible to determine the reflection coefficient for the acoustic wave and amplitude of the slow magnetoacoustic wave propagating downwards. This would be warranted if the downward acoustic wave did not undergo reflection in lower layers of the umbra. However, the Eq. (6), which describes the propagation of acoustic waves in deep umbral layers, and which is well studied in the theory of $p$-mode solar oscillations, indicates that due to the increase of the sound speed with the depth, the downward acoustic wave is totally reflected at some depth and for this reason, the solution of Eq. (6) has the form (9) in sufficiently deep 


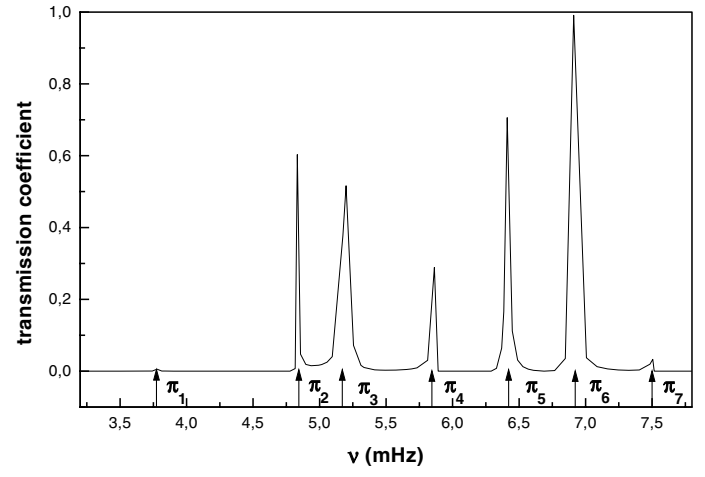

Fig. 5. The transmission coefficient of a slow magnetoacoustic wave. The arrows indicate the real parts of $\pi$-mode frequencies $(k=$ $\left.0,8 \mathrm{Mm}^{-1}, H_{0}=4000 \mathrm{G}\right)$.

layers. This means that the amplitudes of acoustic waves propagating downward and upward should be related at the level $z=z_{i}$ by a certain relation. In this case, however, to satisfy the boundary conditions in the corona (for $z \rightarrow \infty$ ), it has to be assumed that at the level $z=z_{i}$, in addition to the slow magnetoacoustic wave propagating downwards, there also exists a slow wave propagating upwards. In this case the only possible and correct problem is that of the propagation of a slow magnetoacoustic wave through the atmosphere of the umbra; this is the problem considered in this Section.

If we assume that there is no slow magnetoacoustic wave propagating upwards, then the boundary conditions in the corona can be satisfied only at complex frequencies, i.e. in this case we are led to the eigenvalue problem.

Thus, as it was noted above, two approaches can be used to clarify the nature of the sunspot umbral oscillations: to calculate either the eigenvalue spectrum of the oscillations, or the coefficient of transmission of a slow magnetoacoustic wave through the atmosphere of the sunspot umbra. In both cases, the asymptote of the solutions for the system (1) and (2) in deep layers of the umbra should be known.

Some peculiarities of the propagation of a slow magnetoacoustic wave through the atmosphere of the sunspot umbra were considered in the studies of Zhukov (1985) and Zhukov \& Efremov (1988). However, in these studies the approximation of an incompressible medium was used, which is not suitable for high-frequency oscillations. Here, we calculated the transmission coefficient for a slow magnetoacoustic wave without the simplifying assumptions used in the study of Zhukov \& Efremov (1988).

It is shown in Appendix B that in order to find a solution describing the propagation of a slow magnetoacoustic wave, Eq. (B.1) should be integrated with the initial conditions (7).

Figure 5 presents the transmission coefficient of a slow magnetoacoustic wave calculated for the atmosphere of the sunspot umbra described in Appendix A, with the same model parameters as in the study of Zhukov (2002). It follows that the maximum values of the transmission coefficient coincide with the frequencies of $\pi$-modes of the umbral oscillations, i.e. both the transmission and eigenfrequency method yield the same results.
Table 1. Observed and calculated frequencies of umbral ocillations (in $\mathrm{mHz}$ ).

\begin{tabular}{ccc}
\hline \hline Observations & $\mu=0.829$ & $\mu=1.5$ \\
\hline 4.0 & 3.78 & 3.49 \\
4.8 & 4.83 & 5.01 \\
5.2 & 5.20 & 5.35 \\
$5.6,5.7$ & 5.86 & 5.90 \\
6.2 & 6.41 & 6.35 \\
6.7 & 6.91 & 6.78 \\
\hline
\end{tabular}

\section{Discussion}

In the previous sections, we calculated the spectrum of umbral oscillations using both the method of transmission of a slow magnetoacoustic wave through the atmosphere of the umbra, and the derivation of the eigenvalues. It appeared that both methods yielded the same results. Thus we have settled the argument about the preferable way to analyze umbral oscillations and confirmed the concept that 5-min and 3-min umbral oscillations are essentially the same set of $p$-like mode sunspot oscillations (Bogdan 2000).

Here, we used the model of the umbra, in which for the lower layer it was assumed $z_{2}=250 \mathrm{~km}$ and $\mu=0.829$. Although we have used a rather simple model of the sunspot umbra, the calculated frequencies of umbral oscillations are consistent with the observations of Banerjee et al. (2002), presented in Table 1.

The first column of Table 1 presents the frequencies of the oscillations obtained in the study of Banerjee et al. (2002), the second column - the real parts of calculated frequencies $\pi$-modes for $k=0.8 \mathrm{Mm}^{-1}$ and $\mu=0.829$, the third - the real parts of the frequencies also calculated for $k=0.8 \mathrm{Mm}^{-1}$, however, for $\mu=1.5$. Note that in the studies of Brynilndsen et al. $(2002,2004)$ only one peak was found in the power spectrum close to $6.0 \mathrm{mHz}$, while in the study Nindos et al. (2002) two prominent peaks were found at $4.49-5.47 \mathrm{mHz}$ and 6.25-6.45 mHz (see also Rendtel et al. 2003).

Figure $3 \mathrm{a}$ indicates that the $v_{z}$ component of the eigenfunction for the $\pi_{6}$-mode increases with height in the upper layers of the atmosphere of a spot, reaches its maximum in the transition layer and starts to decrease in the lower corona $\left(v_{z}\right.$ components of the other eigenmodes vary with height in the atmosphere of an umbra in a similar way), which qualitatively corresponds to observations made by Brynildsen et al. (2002) and O'Shea et al. (2002).

Until recently, in a theoretical analysis of umbral oscillations it was not considered possible to take properly into account the divergence of magnetic field lines of a spot with height, lateral boundary conditions, non-linear effects, etc. Therefore, it was not possible to expect sufficiently accurate results, such as, for example, those obtained in the theory of $p$-modes of the solar oscillations. However, the latest studies of Rosenthal et al. (2002) and Bogdan et al. (2003) provide hope that these difficulties will be overcome in the near future. 
Acknowledgements. The author thanks the anonymous referees for useful comments and notes.

\section{Appendix A: Model of the sunspot umbra}

Here, as well as in Zhukov (2002), we have studied the following two-layer model of the sunspot umbra:

Layer 1 (Chromosphere-corona, $z>0$ )

$c_{1}^{2}(z)=c_{01}^{2}\left(1+\delta\left[\tanh \left(\alpha\left(\frac{z}{z_{1}}-\beta\right)\right)-\tanh (-\alpha \beta)\right]\right)$,

$\rho_{1}(z)=\rho_{1}(0) \frac{c_{01}^{2}}{c_{1}^{2}(z)}\left(\left(\frac{\xi}{\xi_{0}}\right)^{-\frac{1}{a^{2}}}\left(\frac{\xi^{2}-a^{2}}{\xi_{0}^{2}-a^{2}}\right)^{\frac{a^{2}+1}{2 a^{2}}}\right)^{-\frac{g \gamma z_{1}}{\alpha c_{\infty}^{2}}}$,

where

$\xi=\exp \left(\alpha\left(\frac{z}{z_{1}}-\beta\right)\right), \quad \xi_{0}=\exp (-\alpha \beta)$,

$a^{2}=\frac{\delta-1+\delta \tanh (-\alpha \beta)}{\delta+1-\delta \tanh (-\alpha \beta)}$

and

$c_{\infty}^{2}=c_{01}^{2}[1+\delta(1-\tanh (-\alpha \beta))]$.

Layer 2 (convective zone, $z<0$ )

$c_{2}^{2}(z)=c_{02}^{2}\left(1-\frac{z}{z_{2}}\right)$,

$\rho_{2}(z)=\rho_{2}(0)\left(1-\frac{z}{z_{2}}\right)^{\mu}, \quad \mu=\frac{g \gamma z_{2}}{c_{02}^{2}}-1$.

\section{Appendix B: The fourth-order equation for the horizontal component of velocity}

In order to determine the spectrum of the eigenoscillations of the sunspot umbra one should, in particular, integrate the system (1) and (2), starting from some depth at which the expressions (7) and (8) may be used as the initial conditions. Figure B.1 presents the results of the numerical integration for the system (1) and (2) from $z=-30 z_{2}$ to $z=0$ with the initial conditions (7) and (8). The calculations were carried out for a slow magnetoacoustic wave with the parameters $\omega=0.03 \mathrm{~s}^{-1}$, $k=0.9 \mathrm{Mm}^{-1}$. For lower layers of the sunspot umbra, we used the same parameters as in the study of Zhukov (2002), with the exception that here the magnetic field in the umbra was assumed to be $4000 \mathrm{G}$.

It is apparent that the numerical solution of the system (1) and (2) does not coincide with the asymptotic solution even in deep layers of the sunspot umbra. This means that when the system (1) and (2) is integrated, the initial conditions specified at these depths cannot be restricted by the leading terms of the asymptotes (7) and (8). On the other hand, if the integration is started from substantially greater depths, the solution becomes numerically unstable.

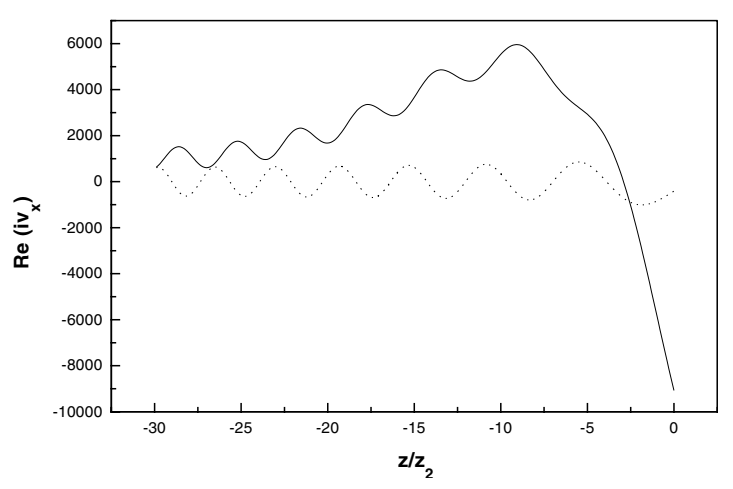

Fig. B.1. The solution for a slow magnetoacoustic wave obtained by the integration of the system (1) and (2) with the initial data (7) and (8) specified for $z=-30 z_{2}$ (solid curve). The dotted curve represents the asymptotic solution (see Eq. (7)).

It may be shown, however, that the system of Eqs. (1) and (2) may be reduced to a single fourth-order differential equation for the horizontal component of the velocity $v_{x}$ :

$\frac{\mathrm{d}^{4} v_{x}}{\mathrm{~d} z^{4}}-A_{3} \frac{\mathrm{d}^{3} v_{x}}{\mathrm{~d} z^{3}}+A_{2} \frac{\mathrm{d}^{2} v_{x}}{\mathrm{~d} z^{2}}-A_{1} \frac{\mathrm{d} v_{x}}{\mathrm{~d} z}+A_{0} v_{x}=0$,

where

$A_{3}=\frac{s}{c^{2}}-\frac{2}{v_{A}^{2}} \frac{\mathrm{d} v_{A}^{2}}{\mathrm{~d} z}+\frac{1}{f} \frac{\mathrm{d} f}{\mathrm{~d} z}+\frac{g}{c^{2}}$,

$$
\begin{aligned}
A_{2}= & \left(\frac{1}{f} \frac{\mathrm{d} f}{\mathrm{~d} z}+\frac{g}{c^{2}}\right)\left(\frac{s}{c^{2}}-\frac{1}{v_{A}^{2}} \frac{\mathrm{d} v_{A}^{2}}{\mathrm{~d} z}\right) \\
& -\frac{1}{v_{A}^{2}} \frac{\mathrm{d}}{\mathrm{d} z}\left(\frac{v_{A}^{2} s}{c^{2}}-\frac{\mathrm{d} v_{A}^{2}}{\mathrm{~d} z}\right)+\frac{f}{c^{2}}+\frac{\omega^{2}}{v_{A}^{2}}-k^{2},
\end{aligned}
$$

$A_{1}=2 k^{2} \frac{1}{v_{A}^{2}} \frac{\mathrm{d} v_{A}^{2}}{\mathrm{~d} z}+\left(\frac{s}{c^{2}}+\frac{1}{f} \frac{\mathrm{d} f}{\mathrm{~d} z}+\frac{g}{c^{2}}\right)\left(\frac{\omega^{2}}{v_{A}^{2}}-k^{2}\right)$,

$$
\begin{aligned}
A_{0}= & \frac{f}{c^{2}} \frac{\omega^{2}-\left(c^{2}+v_{A}^{2}\right) k^{2}}{v_{A}^{2}} \\
& -\frac{1}{v_{A}^{2}} \frac{\mathrm{d}}{\mathrm{d} z}\left[k^{2} \frac{\mathrm{d} v_{A}^{2}}{\mathrm{~d} z}+\frac{s}{c^{2}}\left(\omega^{2}-v_{A}^{2} k^{2}\right)\right] \\
+ & \left(\frac{1}{f} \frac{\mathrm{d} f}{\mathrm{~d} z}+\frac{g}{c^{2}}\right)\left(k^{2} \frac{1}{v_{A}^{2}} \frac{\mathrm{d} v_{A}^{2}}{\mathrm{~d} z}+\frac{s}{c^{2}} \frac{\omega^{2}-v_{A}^{2} k^{2}}{v_{A}^{2}}\right)
\end{aligned}
$$

here

$s=\frac{\mathrm{d} c^{2}}{\mathrm{~d} z}+g(\gamma-1), \quad f=\omega^{2}-\frac{g}{c^{2}} s$.

Figure B.2 presents the results of the numerical integration of the Eq. (B.1) with the initial conditions (7), with the same parameters as those used in the integration of the system (1) and (2). We can see from Fig. B.2 that the numerical solution obtained by the integration of the Eq. (B.1) differs substantially from that described by the asymptotic formula (7) only within a sufficiently thin layer in the vicinity of the minimum temperature. In this sense, a sunspot is a shallow formation. 


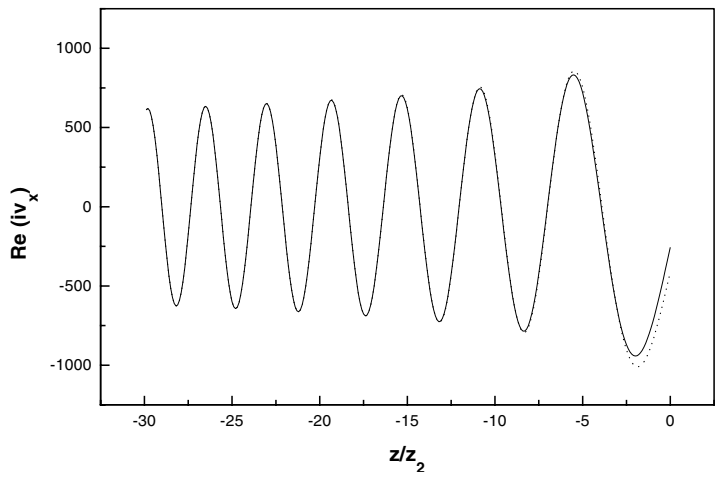

Fig. B.2. The solution for a slow magnetoacoustic wave obtained by integration of the Eq. (B.1) with the initial data (7) specified for $z=-30 z_{2}$ (solid curve). The dotted curve represents the asymptotic solution (see Eq. (7)).

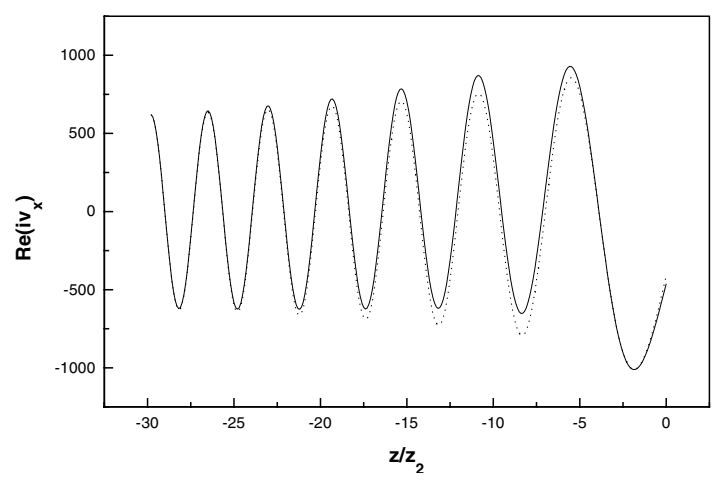

Fig. B.3. The solution for a slow magnetoacoustic wave obtained by the integration of the system (1) and (2) with the initial data specified for $z=-29.9 z_{2}$ and derived from the integration of the Eq. (B.1) (solid curve). The dotted curve represents the asymptotic solution (see Eq. (7)).

Figure B.3 presents the results of the integration of the system (1) and (2) with the initial data obtained by the integration of the Eq. (B.1) from $z=-30 z_{2}$ to $z=-29.9 z_{2}$. It follows that if the integration of the system (1) and (2) is started from moderate depths, then we cannot restrict the initial data only by the primary terms of the asymptotics (7) and (8) (see Fig. B.1). Moreover, the comparison between Figs. B.2 and B.3 indicates that when the same code is used for the numerical integrating, better results are obtained from the integration of the fourth-order equation, rather than the system of two coupled equations.

\section{References}

Banerjee, D., O’Shea, E., Goossens, M., Doyle, J. G., \& Poedts, S. 2002, A\&A, 395, 263

Bogdan, T. J. 2000, Sol. Phys., 192, 373

Bogdan, T. J., \& Cally, P. S. 1997, Proc. R. Soc. London A, 453, 943

Bogdan, T. J., Carlsson, M., Hansteen, V., et al. 2003, ApJ, 599, 626

Brynildsen, N., Maltby, P., Fredvik, T., \& Kjeldseth-Moe, O. 2002, Sol. Phys., 207, 259

Brynildsen, N., Maltby, P., Foley, C. R., Fredvik, T., \& Kjeldseth-Moe, O. 2004, Sol. Phys., 221, 237

Cally, P. S., \& Bogdan, T. J. 1993, ApJ, 402, 721

Cally, P. S., Bogdan, T. J., \& Zweibel, E. G. 1994, ApJ, 437, 505

Evans, D. J., \& Roberts, B. 1990, ApJ, 356, 704

Ferraro, V. C. A., \& Plumpton, C. 1958, ApJ, 127, 459

Gore, A. 1997, Sol. Phys., 171, 239

Gore, A. 1998, Sol. Phys., 178, 13

Gurman, J. B., \& Leibacher, J. W. 1984, ApJ, 283, 859

Hasan, S. S. 1991, ApJ, 366, 328

Hasan, S. S., \& Christensen-Dalsgaard, J. 1992, ApJ, 396, 311

Lee, J. W., \& Yun, H. S. 1987, J. Korean Astron. Soc., 20, 27

Lites, B. W., Thomas, J. H., Bogdan, T. J., \& Cally, P. S. 1998, ApJ, 497, 464

Nindos, A., Alissandrakis, C. E., Gelfreikh, G. B., Bogod, V. M., \& Gontikakis, C. 2002, A\&A, 386, 658

Nye, A. H., \& Thomas, J. H. 1976, ApJ, 204, 582

O'Shea, E., Muglach, K., \& Flek, B. 2002, A\&A, 387, 642

Rendtel, J., Staude, J., \& Curdt, W. 2003, A\&A, 410, 315

Rosenthal, C. S., Bogdan, T. J., Carlsson, M., et al. 2002, ApJ, 564, 508

Scheuer, M. A., \& Thomas, J. H. 1981, Sol. Phys., 71, 21

Settele, A., Zhugzhda, Y. D., \& Staude, J. 1999, Astron. Nachr., 320, 147

Settele, A., Staude, J., \& Zhugzhda, Y. D. 2001, Sol. Phys., 202, 281

Staude, J. 1999, in 3rd Advances in Solar Physics Euroconference Magnetic Fields and Oscillations, ed. B. Schmieder, A. Hofmann, \& J. Staude, ASP Conf. Ser., 184, 113

Thomas, J. H. 1984, A\&A, 135, 188

Thomas, J. H., \& Scheuer, M. A. 1982, Sol. Phys., 79, 19

Uchida, Y., \& Sakurai, T. 1975, Publ. Astron. Soc. Japan, 27, 259

Wood, W. P. 1990, Sol. Phys., 128, 353

Wood, W. P. 1997, Sol. Phys., 173, 259

Zhukov, V. I. 1985, Sol. Phys., 98, 39

Zhukov, V. I. 2002, A\&A, 386, 653

Zhukov, V. I., \& Efremov, V. I. 1988, Bull. Astr. Soc. India, 16, 145

Zhukov, V. I., Efremov, V. I., \& Nuraliev, B. T. 1987, Sol. Phys., 109, 403

Žugžda, Y. D., Locāns, V., \& Staude, J. 1983, Sol. Phys., 82, 369

Žugžda, Y. D., Staude, J., \& Locāns, V. 1984, Sol. Phys., 91, 219

Žugžda, Y. D., Locāns, V., \& Staude, J. 1987, Astron. Nachr., 308, 257 